\title{
Critical appraisal of dabigatran in the treatment of deep vein thrombosis and pulmonary embolism
}

\author{
This article was published in the following Dove Press journal: \\ Journal of Blood Medicine \\ II June 2015 \\ Number of times this article has been viewed
}

\section{Kelechi C Ogbonna Dave L Dixon}

Department of Pharmacotherapy and Outcomes Science, Virginia Commonwealth University School of Pharmacy, Richmond, VA, USA
Correspondence: Kelechi C Ogbonna Department of Pharmacotherapy and Outcomes Science, Virginia Commonwealth University School of Pharmacy, 4I0 North 12th Street, PO Box 980533, Richmond, VA, USA 23298-0533

Tel +l 804628507 I

Fax +l 8048280343

Email kcunegbuogbo@vcu.edu
Objective: To compare the safety and efficacy of dabigatran to warfarin for the treatment of deep vein thrombosis and pulmonary embolism.

Background: Venous thromboembolism (VTE) is a disease comprised of two conditions: deep vein thrombosis and pulmonary embolism. VTE is a major cause of morbidity and mortality worldwide with an annual incidence estimated at $1-3$ cases per 1,000 individuals. This incidence increases with age from 0.1 per 1,000 in adolescence to eight per 1,000 in those 80 years of age and older. As the proportion of patients 65 years of age and older expands, the number of patients presenting with VTE will also increase. Anticoagulation remains the cornerstone of VTE treatment. Traditionally, vitamin $\mathrm{K}$ antagonists have been used to minimize the risk of thrombus extension and for secondary prevention. Unpredictable pharmacokinetics and pharmacodynamics, routine monitoring, drug-food and drug-drug interactions, and potentially severe adverse events have all been cited as barriers to optimal care. Dabigatran has been proposed as a suitable alternative to warfarin therapy in the treatment of VTE. Therefore, a critical appraisal of dabigatran's safety and efficacy is necessary to determine its role in therapy.

Conclusion: Dabigatran remains an alternative to warfarin therapy for the treatment of VTE. However, dabigatran also has distinct disadvantages that warrant consideration. Clinicians must ensure that drug characteristics align with patient characteristics to optimize patient outcomes.

Keywords: venous thromboembolism, pulmonary embolisms, venous thrombosis, anticoagulants, warfarin sodium, dabigatran etexilate mesylate

\section{Introduction}

Venous thromboembolism (VTE) is a disease comprised of two conditions: deep vein thrombosis (DVT) and pulmonary embolism (PE). DVT is a blood clot most often found affecting areas of stasis within the veins of the lower leg or thigh, whereas PE occurs when part of a clot detaches, travels, and lodges in the pulmonary arteries, causing a potentially fatal condition. ${ }^{1}$ VTE is a major cause of morbidity and mortality worldwide with an annual incidence estimated at 1-3 cases per 1,000 individuals. ${ }^{2,3}$ This incidence increases with age from 0.1 per 1,000 in adolescence to eight per 1,000 in those 80 years of age and older. ${ }^{2-4}$ In Europe and the United States, death attributed to VTE is thought to exceed 400,000 annually. 5,6 As the proportion of patients 65 years of age and older expands, the number of patients presenting with VTE will also increase. ${ }^{4}$ Therefore, timely identification and aggressive treatment is essential to reducing morbidity and mortality associated with this disease. 


\section{Risk factors, clinical presentation, and diagnosis}

Several risk factors for developing VTE have been extensively studied and identified (Table 1). ${ }^{7-11}$ Patients with a prior history of VTE and/or those who have cancer are at particularly high risk of developing DVT and progressing to PE. ${ }^{12}$ Likewise, orthopedic procedures, trauma, and hypercoagulability disorders have also been linked to an increased incidence of VTE., ${ }^{412}$ Despite extensive knowledge pertaining to the precipitating factors for VTE, early detection remains challenging.

VTE symptoms are generally nonspecific and often require objective tests for proper diagnosis. ${ }^{13}$ Discomfort in the calf muscle on passive dorsiflexion of the foot, also known as Homans' sign, was once considered the key clinical indicator of DVT. This sign has since fallen out of favor as it is present in less than one-third of patients with confirmed DVT, it is found in more than $50 \%$ of patients without DVT, and it may pose a risk for embolization. ${ }^{14}$ Instead, clinicians should review complaints of leg pain, swelling, edema, or tenderness on palpation. ${ }^{15}$ Other symptoms of DVT may include redness, unexplained fever, increased visibility of skin veins, or bluish discoloration.

Progression to PE occurs in approximately $50 \%$ of untreated proximal DVT cases. ${ }^{12}$ Patients with PE typically present with sudden-onset chest pain, shortness of breath,

\section{Table I VTE risk factors}

Strong risk factors
Fracture (hip or leg)
Hip or knee replacement
Major general surgery
Major trauma
Spinal cord injury
History of VTE
Moderate risk factors
Central venous lines
Chemotherapy
Congestive heart or respiratory failure
Hormone replacement therapy
Cancer
Oral contraceptives
Paralytic stroke
Pregnancy, postpartum
Thrombophilia
Weak risk factors
Bed rest $>3$ days
Increasing age ( $>60$ years)
Obesity (BMI over $30 \mathrm{~kg} / \mathrm{m}^{2}$ )
Pregnancy, antepartum
Varicose veins

Notes: With kind permission from Springer Science+Business Media: Frederick AA Jr, Spencer FA. Risk factors for venous thromboembolism. Circulation. 2003; 107(23 Suppl I):9-16.34

Abbreviations: VTE, venous thromboembolism; BMI, body mass index. tachypnea, and tachycardia. PE is a life-threatening condition and may result in cardiopulmonary collapse. ${ }^{16}$ For these reasons, quick identification of VTE is crucial.

Radiographic contrast studies remain the gold standard for the diagnosis of DVT (venography) and PE (pulmonary angiography). However, their utility in clinical practice is limited. The invasive nature of the studies and need for contrast agents, particularly in critically-ill patients, may pose more risk than benefit. ${ }^{13}$ Radiographic contrast studies are also significantly more expensive than the available alternatives. Less invasive tests, such as compression ultrasound, ventilationperfusion scan, and computed tomography scans are more commonly used in clinical practice for the initial evaluation of suspected VTE. ${ }^{17}$

\section{Treatment strategies}

Anticoagulation remains the cornerstone of VTE treatment. Once the diagnosis of VTE has been confirmed, anticoagulant therapy should begin. Treatment can be divided into two phases: 1) rapid initiation to minimize the risk of thrombus extension; and 2) maintenance for secondary prevention. ${ }^{18}$ Phase 1, or the acute stage of VTE treatment, is generally 7 days and requires rapidly acting anticoagulants such as unfractionated heparin, low-molecular-weight heparin (LMWH), and fondaparinux..$^{15}$ The maintenance phase features continued anticoagulation, traditionally with a vitamin $\mathrm{K}$ antagonist (VKA), intended to reduce the risk of long-term sequelae (ie, post-thrombotic syndrome). ${ }^{15}$ This second phase of therapy allows the body to slowly dissolve the clot via endogenous thrombolytic processes.

The optimal duration of therapy varies. Patients may continue on anticoagulant therapy beyond 3 months, depending on the clot's etiology and site. Patient risk factors pertaining to clotting as well as bleeding will also need to be assessed prior to treatment. ${ }^{15}$ In life- or limb-threatening situations, anticoagulation may be insufficient. Elimination of the obstructing thrombus may be necessary, and the use of thrombolysis or thrombectomy can be considered. ${ }^{15}$

As beneficial as anticoagulants have proven to be in the treatment of VTE, they also come with substantial risk. The anticipation surrounding the development of the targetspecific oral anticoagulants (TSOACs), including dabigatran, rivaroxaban, apixaban, and edoxaban, has stemmed from the challenges that typically accompany VKA therapy. Unpredictable pharmacokinetics and pharmacodynamics, routine monitoring, drug-food and drug-drug interactions, and potentially severe adverse events have all been cited as barriers to optimal care. ${ }^{19}$ Dabigatran has been proposed as a suitable alternative to warfarin therapy in the treatment of 
VTE. A critical appraisal of dabigatran's safety and efficacy is necessary to determine its role in therapy.

\section{Dabigatran characteristics}

Dabigatran was first approved by the European Commission and the US Food and Drug Administration (FDA) to reduce the risk of stroke and systemic embolism in patients with nonvalvular atrial fibrillation in 2008 and 2010, respectively. Dabigatran has since been approved for the treatment and prevention of recurrence of DVT and PE in the USA and Europe, with availability in over 100 countries. Dabigatran is a specific, competitive, and reversible direct thrombin inhibitor. Thrombin enables the conversion of fibrinogen into fibrin during the coagulation cascade. Thus, dabigatran's inhibition of thrombin prevents clot formation. Unlike warfarin, dabigatran only elicits an effect on one factor within the coagulation cascade, possibly allowing for a more predictable pharmacokinetic profile. ${ }^{20}$

\section{Pharmacokinetic and pharmacodynamic properties}

Another differentiating characteristic of dabigatran is its formulation. Formulated as a prodrug, dabigatran etexilate requires hydrolysis for conversion to the active moiety. Dabigatran etexilate is also dependent on an acidic environment for consistent and predictable absorption. For this reason, the drug is applied to a tartaric acid core, which is then encapsulated (Figure 1). The tartaric acid core provides a stable acidic environment, making the formulation less dependent on gastrointestinal acidity.

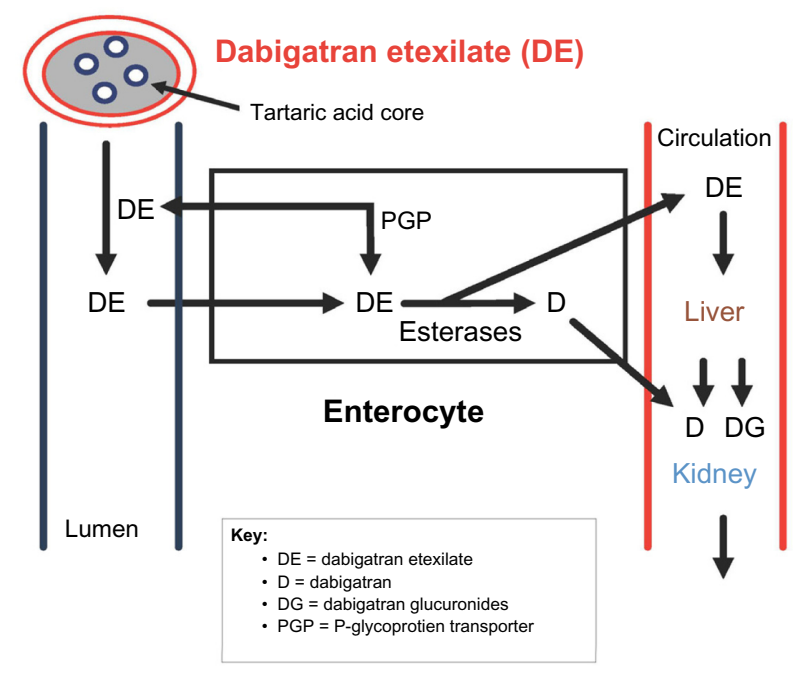

Figure I D characteristics (absorption, distribution, metabolism, excretion). Note: Reproduced from Risk versus benefit of non-vitamin $\mathrm{K}$ dependent anticoagulants compared to warfarin for the management of atrial fibrillation in the elderly. Circulation. 2013;30(7):5 I3-525, Ogbonna KC, Jeffery SM. ${ }^{28}$
Dabigatran has less drug-drug interactions than warfarin; however, drug concentrations can be influenced by the activity of P-glycoprotein transporters. These efflux pumps prevent the absorption of dabigatran etexilate and propel the drug back into the intestinal lumen. Drugs that induce or inhibit P-glycoprotein transporters may affect dabigatran plasma concentrations (Table 2). Finally, dabigatran is primarily excreted via the kidney ( $~ 80 \%)$. As a result, dabigatran exposure is increased in patients with renal impairment, which correlates with the severity of the disease. ${ }^{20,21}$

\section{Special considerations}

The lack of routine monitoring with dabigatran is a distinct advantage over VKAs. However, there are special considerations with dabigatran that are worth noting. Dabigatran has a relatively short half-life ( $\sim 12$ hours) requiring twice-daily dosing. ${ }^{22}$ Therefore, careful attention to adherence with dabigatran is required. Without routine monitoring to assist in identifying nonadherent patients, clinicians will need to educate and inform patients regarding the increased risk of stroke associated with poor adherence. Dabigatran has a quick onset of action as it achieves maximum serum concentration $\left(\mathrm{C}_{\max }\right)$ in 1 hour. ${ }^{22}$ While this can be advantageous, it also means that there is a very short duration of action. This may expose a nonadherent patient to unnecessary risk if not taken properly.

Urgent reversal options for dabigatran include hemodialysis, charcoal, antifibrinolytics, and coagulation factor replacement. ${ }^{23}$ While each of these options has their own respective advantages and disadvantages, the clinical utility of each remains in question. For example, although hemodialysis can decrease dabigatran levels by $60 \%-70 \%$, it requires central venous access. ${ }^{23}$ Fortunately, a reversal agent for dabigatran is in development. Idarucizumab is a humanized monoclonal antibody fragment that has a 350 times higher affinity for

Table 2 Inhibitors and inducers of P-glycoprotein

Inhibitors
Amiodarone
Ketoconazole/itraconazole
Clarithromycin/erythromycin
Verapamil
Diltiazem
Quinidine
Protease inhibitors
Sirolimus/tacrolimus
Inducers
Rifampicin
St John's Wort
Carbamazepine
Phenytoin

Note: Data from Fenner et al..$^{35}$ 
dabigatran than thrombin. Thus far, no immunogenic reactions have been observed. ${ }^{23}$ In early 2015, using interim data from an ongoing Phase III study, ${ }^{24}$ Boehringer Ingelheim (Ingelheim, Germany) submitted applications for approval to the European Medicines Agency, US FDA, and Health Canada. ${ }^{25}$ The US FDA has designated idarucizumab as a "Breakthrough Therapy", which will allow for an accelerated approval pathway. ${ }^{4}$

Dabigatran offers a promising alternative to traditional oral anticoagulant therapy based on its pharmacokinetic and pharmacodynamic characteristics. It is, however, important to evaluate clinical trials investigating the safety and efficacy of dabigatran in the prevention and treatment of VTE.

\section{VTE trial data}

\section{Phase I: acute treatment of VTE}

RE-COVER (number $[\mathrm{n}]=2,564$ ) and RE-COVER II $(n=2,589)$ evaluated dabigatran for the acute treatment of VTE. ${ }^{26,27}$ Both trials used a randomized, double-blind, doubledummy design to compare a fixed dose of dabigatran (150 mg twice daily) with dose-adjusted warfarin (international normalized ratio [INR]: 2.0-3.0) for a 6-month period (Table 3). Initial parenteral anticoagulation was administered for at least 5 days in each study. The primary outcome for both trials was symptomatic and objectively verified recurrent VTE or related death (ie, fatal PE). Dabigatran was found to be noninferior in the reduction of recurrent VTE when compared to warfarin in RE-COVER $(2.4 \%$ vs $2.1 \%$, respectively; hazard ratio $[\mathrm{HR}]=1.10 ; 95 \%$ confidence interval $[\mathrm{CI}]: 0.65-1.84)$ and RE-COVER II (2.3\% vs 2.2\%, respectively; HR =1.08; 95\% CI: 0.64-1.80). ${ }^{26,27}$ Additionally, rates of bleeding were similar between dabigatran and warfarin in each trial (RE-COVER [1.6\% vs $1.9 \%$, respectively; HR $=0.82 ; 95 \%$ CI: $0.45-1.48]$ and RE-COVER II [1.2\% vs $1.7 \%$, respectively; HR $=0.69$; 95\% CI: $0.36-1.32]) .{ }^{26,27}$ While all other adverse effects were similar between the groups, patients receiving dabigatran in the RE-COVER study were more likely to experience dyspepsia compared with patients receiving warfarin $(2.9 \%$ vs $0.6 \%$, respectively; $P<0.001$ ), likely due to its formulation with the tartaric acid core. ${ }^{26,28}$

RE-COVER II was initiated after the completion of the original RE-COVER study due to low rates of recurrent VTE. ${ }^{27}$ The ability to combine the data from both studies allows for robust interpretation and confirmation of the original results of RE-COVER. The additional study provided an expanded pool of subjects that was more in line with the general VTE population with the number of patients with DVT, PE, and both DVT and PE, representing approximately $69 \%, 23 \%$, and $9 \%$, respectively, of the trial population. ${ }^{26,27}$ Pooled analysis of the 5,107 patients from RE-COVER and RE-COVER II maintained noninferiority for the primary efficacy outcome $(2.4 \%$ vs $2.2 \%$, respectively; $\mathrm{HR}=1.09$; 95\% CI: $0.76-1.57)$ and similar rates of major bleeding $(1.4 \%$ vs $2.0 \%$; HR $=0.73 ; 95 \% \mathrm{CI}: 0.48-1.11$ ).

Table 3 Clinical trials with dabigatran in the treatment of VTE

\begin{tabular}{|c|c|c|c|c|}
\hline Trial & RE-COVER & RE-COVER II & RE-MEDY & RE-SONATE \\
\hline \multicolumn{5}{|l|}{ Characteristics } \\
\hline Design & Double-blind; noninferiority & Double-blind; noninferiority & $\begin{array}{l}\text { Double-blind; } \\
\text { noninferiority }\end{array}$ & $\begin{array}{l}\text { Double-blind; } \\
\text { superiority }\end{array}$ \\
\hline $\mathrm{n}$ (patients) & 2,539 & 2,568 & 2,856 & 1,343 \\
\hline Intervention & $\begin{array}{l}\text { Heparin } \geq 5 \text { days followed by } \\
\text { DAB } 150 \mathrm{mg} \text { BID }\end{array}$ & $\begin{array}{l}\text { Heparin } \geq 5 \text { days followed by } \\
\text { DAB I } 50 \mathrm{mg} \text { BID }\end{array}$ & DAB I50 mg BID & $\begin{array}{l}\text { DAB I50 mg } \\
\text { BID }\end{array}$ \\
\hline Control & $\begin{array}{l}\text { Heparin } \geq 5 \text { days and dose- } \\
\text { adjusted warfarin (INR: } 2.0-3.0 \text { ) }\end{array}$ & $\begin{array}{l}\text { Heparin } \geq 5 \text { days and dose- } \\
\text { adjusted warfarin (INR: } 2.0-3.0 \text { ) }\end{array}$ & $\begin{array}{l}\text { Dose-adjusted warfarin } \\
\text { (INR: } 2.0-3.0)\end{array}$ & Placebo \\
\hline Intended duration & 6 months & 6 months & 18 months & 6 months \\
\hline TTR $(\%)$ & 60 & 57 & 64 & NA \\
\hline \multicolumn{5}{|c|}{ Results - efficacy: VTE or VTE-related/unexpected death } \\
\hline $\begin{array}{l}\text { Hazard ratio } \\
(95 \% \mathrm{Cl})\end{array}$ & $\begin{array}{l}1.10 \\
(0.65-1.84)\end{array}$ & $\begin{array}{l}1.08 \\
(0.64-1.80)\end{array}$ & $\begin{array}{l}\text { I.44 } \\
(0.78-2.64)\end{array}$ & $\begin{array}{l}0.08 \\
(0.02-0.25)\end{array}$ \\
\hline$P$-value for & Noninferiority $<0.001$ & Noninferiority $<0.001$ & Noninferiority 0.014 & $\begin{array}{l}\text { Superiority } \\
<0.001\end{array}$ \\
\hline \multicolumn{5}{|c|}{ Results - safety: clinically relevant or major bleeding } \\
\hline $\begin{array}{l}\text { Hazard ratio } \\
(95 \% \mathrm{Cl})\end{array}$ & $\begin{array}{l}0.63 \\
(0.47-0.84)\end{array}$ & $\begin{array}{l}0.62 \\
(0.45-0.84)\end{array}$ & $\begin{array}{l}0.54 \\
(0.4 \mid-0.7 I)\end{array}$ & $\begin{array}{l}2.92 \\
(1.52-5.60)\end{array}$ \\
\hline$P$-value & 0.002 & 0.001 & $<0.001$ & 0.0013 \\
\hline
\end{tabular}

Note: Data from Schulman et al, ${ }^{26}$ Schulman et al, ${ }^{27}$ and Schulman et al. ${ }^{29}$

Abbreviations: VTE, venous thromboembolism; n, number; DAB, dabigatran; BID, twice daily; INR, international normalized ratio; TTR, time in therapeutic range; NA, not applicable; $\mathrm{Cl}$, confidence interval. 


\section{Phase II: extended treatment of VTE}

Extended treatment of VTE with dabigatran was studied in two separate trials, RE-MEDY and RE-SONATE. ${ }^{29}$ RE-MEDY compared the noninferiority of dabigatran $150 \mathrm{mg}$ twice daily vs dose-adjusted warfarin (INR: 2.0-3.0), while RE-SONATE compared the superiority of the same dose of dabigatran vs placebo. Patients in both trials had objectively confirmed symptomatic proximal DVT or PE and completed at least 3 months of treatment with warfarin or dabigatran.

In the RE-MEDY trial, dabigatran demonstrated noninferiority compared with warfarin for the primary efficacy endpoint of recurrent, objectively confirmed, symptomatic VTE or VTE-related death $\left(\mathrm{HR}=1.44 ; 95 \% \mathrm{CI}\right.$ : 0.78-2.64) $($ Table 3$) .{ }^{29}$ Major bleeding was considerably lower with dabigatran when compared with warfarin; however, it did not achieve statistical significance $(P=0.06)$. There was, however, an increased risk of acute coronary syndrome (ACS) in patients randomized to dabigatran $(0.9 \%$ vs $0.2 \% ; P=0.02)$, furthering concerns observed in previous trials. ${ }^{29,30}$

In the RE-SONATE trial (dabigatran vs placebo) dabigatran demonstrated a significant reduction $(92 \%)$ in the primary endpoint of objectively confirmed symptomatic VTE or unexpected death, representing superiority over placebo $(\mathrm{HR}=0.08$; 95\% CI: 0.02-0.25). ${ }^{29}$ While major bleeding alone was not significantly increased with dabigatran, the composite of major or clinically relevant nonmajor bleeding was significantly increased with dabigatran ( $\mathrm{HR}=2.92 ; 95 \% \mathrm{CI}$ : 1.52-5.60). The risk of "any bleeding" was also significantly increased with dabigatran use ( $\mathrm{HR}=1.82$; 95\% CI: 1.23-2.68). Despite the increased risk of ACS observed in the RE-MEDY trial, there was no difference between the two groups in RE-SONATE. This may again speak to the protective effects of warfarin and less so to the harmful effects of dabigatran, as a similar finding was observed in dabigatran's atrial fibrillation studies. ${ }^{27-30}$

\section{Special populations Older adults}

The incidence of VTE and recurrent VTE increases with age and older adults are at a higher risk for death from PE. ${ }^{3}$ Furthermore, the risk of bleeding associated with treatment is higher in this population as well. ${ }^{3}$ The advent of TSOACs is promising in this group of individuals because of the increased potential for drug-drug interactions with warfarin and the need for frequent monitoring. However, dabigatran is highly dependent on renal elimination, which can be problematic considering renal impairment is common in the elderly due to age-related changes in renal function and the high prevalence of chronic kidney disease. This may lead to a greater risk of bleeding in the elderly, particularly those with poor renal function. The use of dabigatran in the elderly is not well established, as this population has been underrepresented in clinical trials. This is especially true for those $\geq 75$ years of age.

The mean age for patients in RE-COVER and RECOVER II was $<60$ years, and only $10.4 \%$ of the population was $\geq 75$ years of age. ${ }^{26,27}$ Despite the small sample of elderly patients, subgroup analyses found no difference between the efficacy of dabigatran and warfarin. However, a meta-analysis found dabigatran to be associated with a greater risk (relative risk $[\mathrm{RR}]=0.9195 \%$ confidence interval $[\mathrm{CI}][0.36-2.26]$ ) of major bleeding in subjects $\geq 75$ years of age compared to apixaban and rivaroxaban $(\mathrm{RR}=0.2395 \% \mathrm{CI}$ [0.08-0.69]; $\mathrm{RR}=0.27\left[0.13-0.59\right.$, respectively.$^{3}$ Although the overall trial results with dabigatran showed a reduced risk of major bleeding with dabigatran when compared to warfarin, there appears to be a greater risk of major bleeding in those $\geq 75$ years of age. Additional studies in this population are warranted to determine the role of dabigatran in the elderly.

\section{Renal impairment}

While dabigatran offer several advantages over warfarin, the safety and efficacy of dabigatran in patients with renal impairment is a concern. Dabigatran significantly depends on renal elimination ( $80 \%$ of total clearance), much more so than the other TSOACs. ${ }^{22}$ Pharmacokinetic data shows a 3.2fold increase in the area under the curve (AUC) for patients with moderate renal impairment (creatinine clearance $[\mathrm{CrCl}]$ : 30-50 $\mathrm{mL} /$ minute), while a 6.3 -fold increase in the AUC was found using pharmacokinetic modeling. ${ }^{22}$ Moderate renal impairment increases the $\mathrm{C}_{\max }$ and half-life minimally, so no additional dose adjustment is recommended for those individuals; however, severe renal impairment has a profound effect on $\mathrm{C}_{\max }$ and half-life. ${ }^{22}$ Of note, this was the basis for the $75 \mathrm{mg}$ twice-daily dose for stroke prophylaxis in patients with atrial fibrillation and a $\mathrm{CrCl}$ of $15-30 \mathrm{~mL} /$ minute, but this dose has not been evaluated in clinical trials. Currently, there are no dosing recommendations for dabigatran when used for VTE prevention or treatment in patients with $\mathrm{CrCl}<30 \mathrm{~mL} /$ minute. ${ }^{22}$ Due to the paucity of data in this subset of patients, the manufacturer recommends avoiding dabigatran in patients with $\mathrm{CrCl}<30 \mathrm{~mL} /$ minute. $^{22}$

Although individuals with severe renal impairment ( $\mathrm{CrCl}<30 \mathrm{~mL} /$ minute) have been excluded from clinical trials with all of the TSOACs, we can draw some fundamental conclusions from enrolled subjects with mild-moderate renal impairment. A subgroup analysis of subjects with reduced renal function $(\mathrm{CrCl} \leq 50 \mathrm{~mL} /$ minute $)$ found a lower risk of 
VTE recurrence with dabigatran compared to warfarin, but this was not statistically significant $(\mathrm{RR}=0.11 ; 95 \% \mathrm{CI}$ : $0.01-1.88){ }^{3}$ Of note, apixaban and rivaroxaban appear to be associated with an increased risk in recurrence in patients with reduced renal function. ${ }^{3}$ Despite the small sample, the risk of major bleeding was 7.2 times higher in subjects with a $\mathrm{CrCl} \leq 50 \mathrm{~mL} /$ minute compared to subjects with a $\mathrm{CrCl}>50 \mathrm{~mL} /$ minute. $^{3}$ Note the risk of major bleeding with warfarin was only 2.9 times higher in those with a $\mathrm{CrCl} \leq 50 \mathrm{~mL} /$ minute. $^{3}$

\section{Cancer}

Cancer is an independent risk factor for VTE and 20\% of all VTEs occur in individuals with cancer. ${ }^{31}$ The pathophysiology is multifactorial, often involving pre-existing risk factors (eg, obesity, age) and cancer-specific risk factors (eg, the type of cancer, chemotherapy). The recommended long-term treatment for VTE in cancer patients is LMWH because of its superior efficacy over warfarin. ${ }^{32}$ However, not all patients are candidates for LMWH, especially if they have severe renal impairment or if it is the patient's preference not to perform daily injections. While warfarin is a reasonable alternative, it has many disadvantages. Thus, the TSOACs are appealing since these agents are administered orally instead of via subcutaneous injection.

None of the TSOACs, including dabigatran, have been studied exclusively in patients with active cancer. Those with cancer were, however, allowed to enroll in RE-COVER and RE-COVER II, but only $8 \%$ of the population from each trial had cancer. ${ }^{26,27}$ Furthermore, the eligibility criteria for patients with cancer were relatively broad and included patients with recurrent/metastatic cancer, a cancer diagnosis, or any treatment for cancer, in the 5 years preceding study enrollment. RE-MEDY and RE-SONATE also had similar numbers of patients with active cancer at baseline. ${ }^{29}$ Although there were no differences between dabigatran and warfarin in the subgroups of cancer patients for each of these trials, no concrete conclusions can be made due to the small number of patients. Additionally, warfarin is not the preferred option in patients with cancer, so it is unknown how dabigatran would compare with LMWH. ${ }^{32}$ Current guidelines do not recommend any of the TSOACs for either the prevention or treatment of VTE in patients with cancer. ${ }^{32}$ Future studies are warranted and should compare the efficacy and safety of dabigatran with LMWH since it is now the standard of care.

\section{Role in therapy}

Dabigatran has similar efficacy when compared to warfarin for the treatment of VTE. Although dabigatran offers convenience and fewer drug-drug interactions when compared to warfarin, these attributes are offset by adherence concerns and the lack of a reversal agent. Utilization in special populations must also be carefully considered, as dabigatran's use in renal impairment, cancer, and for patients $>65$ years of age requires further investigation.

Dabigatran's bleeding profile is comparable to that of warfarin; however, key differences exist. For example, dabigatran is associated with a lower incidence of intracranial hemorrhage; this may be offset by the increased rate of gastrointestinal bleeding and concern for ACS. In the first quarter of 2011, the Institute for Safe Medication Practices (ISMP) identified 932 serious adverse drug events attributed to dabigatran. ${ }^{33}$ Of the 932 adverse drug events identified, 293 were further classified as gastrointestinal hemorrhage. The ISMP elaborated further by highlighting elderly patients' predisposition to hemorrhagic complications with dabigatran therapy. The median age for dabigatran case reports during this quarter was 80 years compared to a median age of 56 years for all other drugs, ${ }^{33}$ further emphasizing the need for assessment of individual patient characteristics and preexisting conditions prior to therapy selection.

Another disadvantage is the need for parenteral anticoagulation for 5-10 days before dabigatran initiation. Rivaroxaban and apixaban have both been studied as an initial treatment for acute VTE without the need to wait 5 days prior to starting therapy. This caveat to dabigatran therapy may dissuade its use in the acute treatment of VTE.

\section{Conclusion}

Dabigatran remains an alternative to warfarin for the treatment of VTE. Selection of this therapy may be appropriate for those patients unable to tolerate the requirements of warfarin management. Patient characteristics, as well as cost, will need to be considered on a case-by-case basis. The therapeutic armament for the treatment of VTE has increased considerably over the last 5 years. Despite the available options, clinicians must ensure that the drug characteristics align with patient characteristics to optimize patient outcomes.

\section{Disclosure}

The authors report no conflicts of interest in this work.

\section{References}

1. Weill-Engerer S, Meaume S, Lahlou A, et al. Risk factors for deep vein thrombosis in inpatients aged 65 and older: a case-control multicenter study. JAm Geriatr Soc. 2004;52(8):1299-1304.

2. Bramlage P, Pittrow D, Kirch W. Current concepts for the prevention of venous thromboembolism. Eur J Clin Invest. 2005;35 Suppl 1:4-11. 
3. Geldhof V, Vandenbriele C, Verhamme P, Vanassche T. Venous thromboembolism in the elderly: efficacy and safety of non-VKA oral anticoagulants. Thromb J. 2014;12:21.

4. Reitsma PH, Versteeg HH, Middeldorp S. Mechanistic view of risk factors for venous thromboembolism. Arterioscler Thromb Vasc Biol. 2012;32(3):563-568

5. Cohen AT, Agnelli G, Anderson FA, et al; VTE Impact Assessment Group in Europe (VITAE). Venous thromboembolism (VTE) in Europe. The number of VTE events and associated morbidity and mortality. Thromb Haemost. 2007;98(4):756-764.

6. Deitelzweig SB, Johnson BH, Lin J, Schulman KL. Prevalence of clinical venous thromboembolism in the USA: current trends and future projections. Am J Hematol. 2011;86(2):217-220.

7. Giannakopoulos B, Passam F, Rahgozar S, Krilis SA. Current concepts on the pathogenesis of the antiphospholipid syndrome. Blood. 2007;109(2):422-430.

8. Rosendaal FR, Helmerhorst FM, Vandenbroucke JP. Female hormones and thrombosis. Arterioscler Thromb Vasc Biol. 2002;22(2):201-210.

9. Gould MK, Garcia DA, Wren SM, et al; American College of Chest Physicians. Prevention of VTE in nonorthopedic surgical patients: Antithrombotic Therapy and Prevention of Thrombosis, 9th ed: American College of Chest Physicians Evidence-Based Clinical Practice Guidelines. Chest. 2012;141(2 Suppl):e227S-e277S.

10. Kahn SR, Lim W, Dunn AS, et al; American College of Chest Physicians. Prevention of VTE in nonsurgical patients: Antithrombotic Therapy and Prevention of Thrombosis, 9th ed: American College of Chest Physicians Evidence-Based Clinical Practice Guidelines. Chest. 2012;141(2 Suppl):e195S-e226S.

11. Falck-Ytter Y, Francis CW, Johanson NA, et al; American College of Chest Physicians. Prevention of VTE in orthopedic surgery patients: Antithrombotic Therapy and Prevention of Thrombosis, 9th ed: American College of Chest Physicians Evidence-Based Clinical Practice Guidelines. Chest. 2012;141(2 Suppl):e278S-e325S.

12. Kearon C. Natural history of venous thromboembolism. Circulation. 2003;107(23 Suppl 1):I22-I30.

13. Bates SM, Jaeschke R, Stevens SM, et al; American College of Chest Physicians. Diagnosis of DVT: Antithrombotic Therapy and Prevention of Thrombosis, 9th ed: American College of Chest Physicians Evidence-Based Clinical Practice Guidelines. Chest. 2012; 141(2 Suppl):e351S-e418S.

14. Gorman WP, Davis KR, Donnelly R. ABC of arterial and venous disease. Swollen lower limb-1: general assessment and deep vein thrombosis. BMJ. 2000;320(7247):1453-1456.

15. Kearon C, Akl EA, Comerota AJ, et al; American College of Chest Physicians. Antithrombotic therapy for VTE disease: Antithrombotic Therapy and Prevention of Thrombosis, 9th ed: American College of Chest Physicians Evidence-Based Clinical Practice Guidelines. Chest. 2012;141(2 Suppl):e419S-e494S.

16. Tapson VF. Acute pulmonary embolism. N Engl J Med. 2008;358(10): 1037-1052.

17. Mos IC, Klok FA, Kroft LJ, de Roos A, Huisman MV. Imaging tests in the diagnosis of pulmonary embolism. Semin Respir Crit Care Med. 2012;33(2):138-143.

18. Goldhaber SZ, Bounameaux H. Pulmonary embolism and deep vein thrombosis. Lancet. 2012;379(9828):1835-1846.

19. Dobesh PP, Fanikos J. New oral anticoagulants for the treatment of venous thromboembolism: understanding differences and similarities. Drugs. 2014;74(17):2015-2032.

20. Stangier J, Rathgen K, Stähle H, Gansser D, Roth W. The pharmacokinetics, pharmacodynamics and tolerability of dabigatran etexilate, a new oral direct thrombin inhibitor, in healthy male subjects. Br J Clin Pharmacol. 2007;64(3):292-303.
21. Stangier J, Rathgen K, Stähle H, Mazur D. Influence of renal impairment on the pharmacokinetics and pharmacodynamics of oral dabigatran etexilate: an open-label, parallel-group, single-centre study. Clin Pharmacokinet. 2010;49(4):259-268.

22. Pradaxa $^{\circledR}$ (dabigatran etexilate mesylate) capsules [package insert] Ridgefield, CT: Boehringer Ingelheim Pharmaceuticals Inc.; 2015.

23. Mo Y, Yam FK. Recent advances in the development of specific antidotes for target-specific oral anticoagulants. Pharmacotherapy. 2015;35(2):198-207.

24. Boehringer Ingelheim. Reversal of dabigatran anticoagulant effect with idarucizumab. Available from: https://clinicaltrials.gov/ct2/show/ NCT02104947. NLM identifier: NCT02104947. Accessed March 23 2015.

25. Boehringer Ingelheim. Boehringer Ingelheim submits applications for approval of idarucizumab*, specific reversal agent to dabigatran etexilate $\left(\right.$ Pradaxa $\left.^{\circledR}\right)$, to EMA, FDA and Health Canada [press release]. Ingelheim, Germany: Boehringer Ingelheim; 2015 [March 3]. Available from: https://www.boehringer-ingelheim.com/news/news_releases/ press_releases/2015/03_march_2015_dabigatranetexilate.html. Accessed March 23, 2015.

26. Schulman S, Kearon C, Kakkar AK, et al; RE-COVER Study Group. Dabigatran versus warfarin in the treatment of acute venous thromboembolism. N Engl J Med. 2009;361(24):2342-2352.

27. Schulman S, Kakkar AK, Goldhaber SZ, et al; RE-COVER II Trial Investigators. Treatment of acute venous thromboembolism with dabigatran or warfarin and pooled analysis. Circulation. 2014;129(7):764-772.

28. Ogbonna KC, Jeffery SM. Risk versus benefit of non-vitamin K dependent anticoagulants compared to warfarin for the management of atrial fibrillation in the elderly. Drugs Aging. 2013;30(7):513-525.

29. Schulman S, Kearon C, Kakkar AK, et al; RE-MEDY Trial Investigators; RE-SONATE Trial Investigators. Extended use of dabigatran, warfarin, or placebo in venous thromboembolism. $N$ Engl $\mathrm{J} \mathrm{Med}$. 2013;368(8):709-718.

30. Connolly SJ, Ezekowitz MD, Yusuf S, et al; RE-LY Steering Committee and Investigators. $N$ Engl J Med. 2009;361(12):1139-1151.

31. Laporte S, Mismetti P, Décousus H, et al; RIETE Investigators. Clinical predictors for fatal pulmonary embolism in 15,520 patients with venous thromboembolism: findings from the Registro Informatizado de la Enfermedad TromboEmbolica venosa (RIETE) Registry. Circulation. 2008;117(13):1711-1716.

32. Lyman GH, Khorana AA, Kuderer NM, et al; American Society of Clinical Oncology Clinical Practice. Venous thromboembolism prophylaxis and treatment in patients with cancer: American Society of Clinical Oncology clinical practice guideline update. J Clin Oncol. 2013;31(17):2189-2204.

33. Institute for Safe Medication Practices. QuarterWatch. Monitoring FDA MedWatch Reports: Signals for Dabigatran and Metoclopramide. Horsham, PA: Institute for Safe Medication Practices; 2012. Available from: http://www.ismp.org/QuarterWatch/pdfs/2011Q1.pdf. Accessed April 20, 2015.

34. Frederick AA Jr, Spencer FA. Risk factors for venous thromboembolism. Circulation. 2003;107(23 Suppl I):9-16.

35. Fenner KS, Troutman MD, Kempshall S et al. Drug-drug interactions mediated through P-glycoprotein: clinical relevance and in vitro-in vivo correlation using digoxin as a probe drug. Clin Pharmacol Ther. 2009;85(2):173-181. 
Journal of Blood Medicine

\section{Publish your work in this journal}

The Journal of Blood Medicine is an international, peer-reviewed, open access, online journal publishing laboratory, experimental and clinical aspects of all topics pertaining to blood based medicine including but not limited to: Transfusion Medicine; Blood collection, Donor issues, Transmittable diseases, and Blood banking logistics; Immunohematology; Artificial and alternative

Submit your manuscript here: http://www.dovepress.com/Journal-of-blood-medicine-journal

blood based therapeutics; Hematology; Biotechnology/nanotechnology of blood related medicine; Legal aspects of blood medicine; Historical perspectives. The manuscript management system is completely online and includes a very quick and fair peer-review system. Visit http://www.dovepress.com/ testimonials.php to read real quotes from published authors. 